that were more abundant in IBD than controls (propan-1-ol and phenol) returned to levels similar to controls following treatment (figure 1).

Within IBD, the subtype (CD versus colitis (UC and IBDunclassified)) described a small amount of variation (3\%, $\mathrm{p}=0.006)$, with three faecal VOCs (6-methylhept-5-en-2-one; benzaldehyde; 4-methylphenol) significantly different in abundance between CD and colitis (t-test, $\mathrm{p}<0.05$ ).

Conclusion/Interpretation Characterisation of faecal VOCs may advance the understanding of the pathogenesis of IBD, disease sub-types and response to treatment.

\section{P86 PERMANENT STOMA FORMATION IN CROHN'S DISEASE IS ASSOCIATED WITH INCREASED RATES OF ANTIDEPRESSANT USE}

1,2,3 Jonathan Blackwell ${ }^{*}$ 2Prof Sonia Saxena, 1,2,3 Nishani la ${ }^{4}$ Christopher Alexakis, ${ }^{6}$ Matthew Hotopf, ${ }^{1,3}$ Richard Pollok. ${ }^{1}$ St George's University of London, UK; ${ }^{2}$ Imperial College London, UK; ${ }^{3}$ St George's Hospitals NHS Foundation Trust, Dept Gastroenterology, London, UK; ${ }^{4}$ Royal Surrey Hospital, Guildford, UK; ${ }^{5}$ University College London, UK; ${ }^{6}$ Institute of Psychiatry Psychology and Neuroscience, King's College London, UK

\subsection{6/gutjnl-2020-bsgcampus. 161}

Introduction $50 \%$ of patients with Crohn's Disease (CD) will require surgery within the first 10 years after being diagnosed. The impact of having a temporary or permanent stoma on mental health in IBD is unknown.

Aim To examine the impact of intestinal surgery and stoma formation on antidepressant medication (ADM) use.

Methods Using the Clinical Practice Research Datalink, a nationally representative research database, we identified patients with $\mathrm{CD}$ who underwent their first intestinal surgery between 1998-2018. We identified all prescriptions for the 7 most commonly prescribed antidepressant medications: escitalopram, sertraline, citalopram, fluoxetine, paroxetine, venlafaxine, and mirtazapine. Tricyclic antidepressants were excluded since we have previously found they are rarely used for mood disorders and given at low dose for other conditions. Patients were excluded if they had a prescription for an ADM in the 6 months before surgery. Those undergoing intestinal surgery were stratified into three patient groups: without a stoma, temporary stoma, and permanent stoma. We used survival analysis to generate Kaplan-Meier curves to

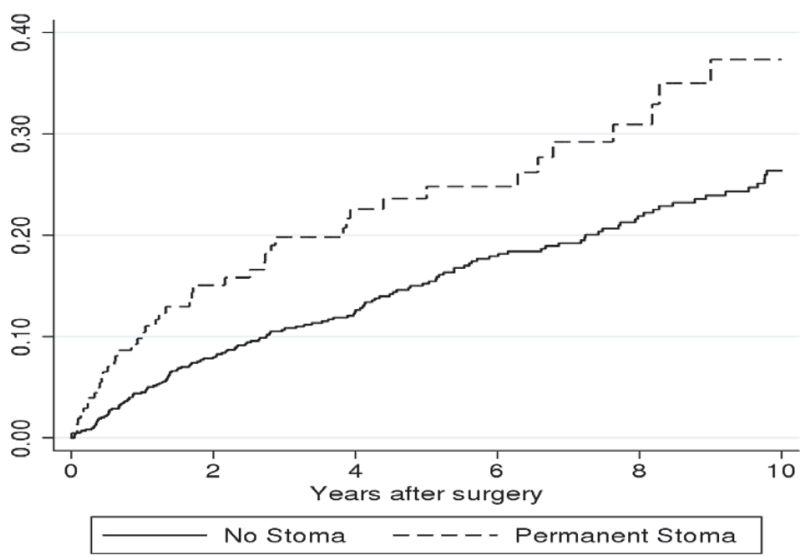

Abstract P86 Figure 1 Risk of antidepressant medication use after first intestinal surgery stratified by stoma status estimate the risk of ADM use in the 10 years after intestinal surgery. We used multiple Cox regression to identify risk factors for ADM use after intestinal surgery. We adjusted for the following covariates within the regression model: sex, age at surgery, smoking status, socio-economic status (index of multiple deprivation) and early surgery within the first year of diagnosis.

Results We identified 1,367 cases of CD undergoing their first intestinal surgery. $71 \%$ did not have a stoma ( $n=974), 14 \%$ had a temporary stoma $(n=190)$, and $15 \%$ received a permanent stoma $(n=203)$. The 10-year risk of ADM use in each group was $26.4 \%, 33.4 \%$ and $37.3 \%$ respectively. Patients with a permanent stoma were $67 \%$ more likely to require an ADM than patients undergoing intestinal surgery without a stoma (HR 1.67, 95\% CI 1.15-2.42, Abstract P86 figure 1). Patients with a temporary stoma had a similar risk of requiring an $\mathrm{ADM}$ to patients undergoing intestinal surgery without stoma formation (HR 1.18, 95\% CI 0.82-1.68).

Conclusion Permanent stoma formation is associated with significantly increased ADM use after intestinal surgery and is like to be associated with increased anxiety and depression. More research to understand how having a stoma impacts on mental health is warranted.

\section{P87 THE IBD REGISTRY AS A PLATFORM FOR STEROID THERAPY AUDIT: TIME TRENDS IN TREATMENT DURATION}

${ }^{1}$ Mustafa Shawihdi ${ }^{*},{ }^{2}$ Sarah Miles, ${ }^{2}$ Liz Dobson, ${ }^{2,3}$ Fraser Cummings, ${ }^{2,4}$ Stuart Bloom, ${ }^{2}$ Stephen Grainger, ${ }^{1,2,5}$ Keith Bodger. 'University Of Liverpool, Liverpool, UK; ${ }^{2} U K$ IBD Registry, UK; ${ }^{3}$ University Hospitals Southampton, Southampton, UK; ${ }^{4}$ University College London Hospital, London, UK; ${ }^{5}$ Aintree University Hospital, UK

\subsection{6/gutjnl-2020-bsgcampus. 162}

Introduction Oral corticosteroids continue to play a key role in inducing remission in inflammatory bowel disease (IBD) but are not effective as maintenance agents. Avoiding prolonged courses of treatment is an important strategy to minimise side effects. The UK IBD Registry has established an infrastructure capable of recording prescribing at point-ofcare using a range of different local systems and software, including direct capture from local operational records. We investigated the feasibility of extracting data to undertake audit of steroid prescribing for hospitals participating in the Registry.

Methods Data submitted to the UK IBD Registry were analysed over three consecutive fiscal years (2016/17, 2017/18 and 2018/19). All prescriptions for oral steroids (prednisolone or budesonide) with a record of both a start and stop date were extracted and linked to patient characteristics. We compared the mean duration of steroid courses initiated in each year and the proportion lasting eight weeks or less.

Results There were 2,156 prescriptions (prednisolone 83\%; budesonide 17\%) with a start and stop date, relating to 1,591 patients treated at 42 hospitals. Three quarters $(77.4 \%)$ of cases had a single steroid prescription recorded over the three year time period $(5.8 \%$ had $>2)$. Results are summarised in the table 1 . There was a year-on-year reduction in the mean duration of recorded steroid courses from 13.0 to 8.4 weeks $(\mathrm{p}<0.01$, ANOVA).

Conclusion IBD teams participating in the UK IBD Registry have achieved a reduction in the duration of steroid treatment courses, suggesting progress in efforts to avoid prolonged 